\title{
Prevalence and Characterization of Thyroid Hemiagenesis in Japan: The Fukushima Health Management Survey
}

\author{
Satoru Suzuki, ${ }^{1,2}$ Sanae Midorikawa, ${ }^{2,3}$ Takashi Matsuzuka, ${ }^{2,4}$ Toshihiko Fukushima, ${ }^{5}$ Yuko Ito, ${ }^{2,6}$ \\ Hiroki Shimura, ${ }^{2,6}$ Hideto Takahashi, Tetsuya Ohira, ${ }^{2,7}$ Akira Ohtsuru, ${ }^{2,3}$ \\ Masafumi Abe, Shinichi Suzuki, ${ }^{2,5}$ and Shunichi Yamashita ${ }^{2,8}$
}

Background: Thyroid hemiagenesis is a rare congenital variant characterized by the lack of development of one thyroid lobe with no clinical manifestations.

Methods: This study was performed to determine the prevalence and characteristics of thyroid hemiagenesis in a normal Japanese population. This cross-sectional study was performed from October 9, 2011, to April 30, 2015. In total, 299,908 children and young adults in the Fukushima Health Management Survey were examined to determine the presence of thyroid agenesis or hemiagenesis. Thyroid width, thickness, and length were measured in 292,452 of these subjects.

Results: Thyroid agenesis was diagnosed in 13 subjects, and hemiagenesis was detected in 67 subjects $(0.02 \% ; 22.3 /$ 100,000 individuals). Although there was no significant sex-related difference $(p=0.067)$, the female:male ratio was 1.67:1.00. Females were significantly dominant in right hemiagenesis, while there was no difference in left hemiagenesis between males and females. The thyroid volumes at the 2.5th and 97.5th percentiles for age and body surface area were determined for each sex. Multivariate regression analysis showed that a large hemithyroid volume was independently associated with the presence of contralateral hemiagenesis $(p<0.001)$.

Conclusion: The prevalence of thyroid hemiagenesis in the present study is in agreement with that reported in other countries. The prevalence of right hemiagenesis was higher in females, and the larger contralateral lobe in patients with rather than without hemiagenesis may have been caused by a compensatory feedback mechanism to prevent hypothyroidism. In addition, the prevalence of hemiagenesis, especially right hemiagenesis, may be affected by sex-related factors similar to those in patients with an ectopic thyroid gland.

Keywords: thyroid hemiagenesis, ultrasonography, health survey

\section{Introduction}

$\mathbf{T}$ HyRoid hemiagenesis is a RARE congenital variant characterized by the lack of development of one thyroid lobe with no clinical manifestations. Because neither clinical symptoms nor signs are present, this variant may be incidentally detected during ultrasound screening of the neck in patients suspected to have thyroid- or other neck-associated disorders or otolaryngological disorders.

Handfield-Jones (1) reported the first case of thyroid hemiagenesis in 1866. Since then, numerous studies involving autopsies, postmortem examinations, and surgical cases have been performed (2). The first reported prevalence of thyroid hemiagenesis detected by systemic ultrasound examination in normal children and young adults was $0.2 \%$ in a study conducted in Belgium (3).

After the Fukushima Daiichi Nuclear Power Plant accident following the Great East Japan Earthquake in 2011, the Fukushima prefectural government initiated the Fukushima Health Management Survey, which involved mass ultrasonographic thyroid screening. A request was made to clarify the existence of effects of low-level radiation exposure such as thyroid abnormalities, especially thyroid cancer, in Fukushima Prefecture (4). This request was based on the significant

\footnotetext{
${ }^{1}$ Department of Thyroid and Endocrinology, Division of Internal Medicine; ${ }^{2}$ Radiation Medical Science Center for the Fukushima Health Management Survey; Departments of ${ }^{3}$ Radiation Health Management, ${ }^{4}$ Otolaryngology, ${ }^{5}$ Thyroid and Endocrinology, ${ }^{6}$ Laboratory Medicine, and ' Epidemiology, School of Medicine; Fukushima Medical University Hospital, Fukushima, Japan.

${ }^{8}$ Department of Radiation Medical Sciences, Atomic Bomb Disease Institute, Nagasaki University, Nagasaki, Japan.

(c) Satoru Suzuki et al. 2017; Published by Mary Ann Liebert, Inc. This article is available under the Creative Commons License CC-BY-NC (http://creativecommons.org/licenses/by-nc/4.0). This license permits non-commercial use, distribution and reproduction in any medium, provided the original work is properly cited. Permission only needs to be obtained for commercial use and can be done via RightsLink.
} 
increase in cases of radiation-associated thyroid cancer following the Chernobyl accident in 1986 (5). Throughout the course of the study, approximately 70 patients with asymptomatic thyroid deformity were identified. No reports to date have mentioned either the prevalence of thyroid hemiagenesis in Japan or the presence/absence of hemiagenesis among nearly 300,000 subjects systematically estimated worldwide. This study describes the difference in the occurrence of thyroid hemiagenesis between the right and left sides and between sexes, as well as the prevalence of hemiagenesis in Japan. It also provides statistical evidence of enlargement of the intact thyroid lobe in patients with hemiagenesis using reference thyroid volume parameters to substantiate the presence of compensatory hypertrophy of the thyroid gland.

\section{Materials and Methods}

\section{Primary thyroid ultrasound examination}

The thyroid ultrasound examination of the Fukushima Health Management Survey was performed on approximately 360,000 subjects who were living in Fukushima Prefecture at the time of the accident and were aged $\leq 18$ years on March 11, 2011, as previously reported (6). In brief, the participation rate was $80.2 \%$ for subjects aged $0-23$ years at the time of the first baseline survey. An ultrasound volumetric examination was performed using a commercial ultrasound machine equipped with a linear array probe $(10-12 \mathrm{MHz})$, and all subjects were evaluated for the presence thyroid nodules and cysts (7). The width, thickness, and height of each lobe were measured, and the volume of each lobe was calculated using the mean elliptical shape volume, determined as follows: $\pi / 6 \times$ width $\times$ thickness $\times$ height. In addition, any extra- or intrathyroidal ultrasound abnormalities (e.g., dysgenesis of the thyroid, lymph node swelling, or enlargement of the intrathyroidal thymus, ultimobranchial body, and/or thyroid) were detected. The frequencies of thyroid agenesis and hemiagenesis were calculated among 299,908 subjects. Some data were not obtained for 3044 subjects because of difficulty performing the thyroid measurements. In addition, the quality of either the height or weight measurement was unsatisfactory for 4412 subjects; these individuals were eliminated from the study. In total, 292,452 subjects (147,575 males) were completely examined and underwent thyroid volume assessment. The subjects with thyroid hemiagenesis were subsequently compared to normal subjects. The normal thyroid volume for each sex and age was calculated using data from all 292,452 subjects after excluding 79 subjects with agenesis or hemiagenesis with complete determination of the body surface area. The body surface area was calculated by the DuBois formula (8). The current study was approved by the Ethics Committee of
Fukushima Medical University (No. 1318). Written informed consent was obtained from all surveyed subjects or the parents of subjects $<18$ years of age. Brief medical records, such as data regarding medications, medical history, and family history, were obtained by a questionnaire. The raw data used to create all tables in the present study are unavailable due to a restriction outlined in the informed consent agreement.

\section{Statistical analysis}

Fisher's exact test for categorical variables was used to compare differences in right, left, and right + left (total) hemiagenesis between the two sexes using IBM SPSS Statistics for Windows v19 (IBM Corp., Armonk, NY; Table 1). The difference in the thyroid volume was analyzed using analysis of covariance (ANCOVA). All $p$-values refer to ANCOVA, adjusted for sex, age, and sex- and age-specific body surface area. Because the body surface area was closely associated with age at the time of surveying (Pearson's correlation coefficient $=0.918$ ), sex and age adjustments were applied for body surface area using a residual model when performing all analyses. Further, because the body surface area was closely associated with the body mass index (Pearson's correlation coefficient $=0.734$ ), the body mass index was eliminated as a covariate. Multivariate regression analysis was used to determine whether sex, age, sex- or age-specific body surface area, or the presence/absence of hemiagenesis independently affected the contralateral hemithyroid volume.

\section{Results}

Prevalence of thyroid agenesis as determined by thyroid ultrasound examination

Among all 299,908 subjects, 13 (12 female) had agenesis of both thyroid lobes. All 13 subjects were taking medication or receiving routine checkups for thyroid function at the time of the survey.

\section{Prevalence of thyroid hemiagenesis characterized by laterality}

As shown in Table 1, $67(0.02 \%)$ subjects had left or right hemiagenesis. The prevalence of right hemiagenesis (12 subjects) was significantly lower than that of left hemiagenesis (55 subjects; $p<0.001)$.

\section{Prevalence of thyroid hemiagenesis characterized by sex}

The female:male ratio was 1.67:1.00, although there was no significant difference $(p=0.067)$. Specifically, right hemiagenesis was diagnosed in 12 subjects, 10 of whom were

Table 1. Prevalence of Thyroid Hemiagenesis by Sex and Laterality

\begin{tabular}{|c|c|c|c|c|c|c|c|c|c|c|}
\hline & \multirow{3}{*}{$\begin{array}{c}\text { Number of } \\
\text { subjects }\end{array}$} & \multicolumn{6}{|c|}{ Hemiagenesis } & & & \\
\hline & & \multicolumn{3}{|c|}{ Right } & \multicolumn{3}{|c|}{ Left } & \multicolumn{3}{|c|}{ Number of subjects with hemiagenesis } \\
\hline & & $\mathrm{n}$ & $\%$ & $\mathrm{p}$ & $\mathrm{n}$ & $\%$ & $\mathrm{p}$ & $\mathrm{n}$ & $\%$ & $\mathrm{p}$ \\
\hline Female & 148,534 & 10 & 0.007 & 0.021 & 31 & 0.021 & 0.346 & 41 & 0.028 & 0.067 \\
\hline Male & 151,374 & 2 & 0.001 & & 24 & 0.016 & & 26 & 0.017 & \\
\hline Totals & 299,908 & 12 & 0.004 & & 55 & 0.018 & & 67 & 0.022 & \\
\hline
\end{tabular}


female. The female:male ratio was 5.4:1.0, with a significant difference $(p=0.021)$. In total, $31 / 55$ subjects with left hemiagenesis were female, and the female:male ratio was 1.3:1.0. There was no significant difference $(p=0.346)$ between the two sexes. Ectopic thyroid tissue was not detected by ultrasound in any subjects.

\section{Thyroid volume by sex and age as measured by ultrasound}

The box-and-whisker plot in Figure 1 shows the 2.5th, 25th, 50th, 75th, and 97.5th percentiles of the thyroid volume according to sex and age. Supplementary Tables S1 and S2 show the 2.5th and 97.5th percentiles of width, thickness, height, and volume for each sex according to age and body surface area, respectively (Supplementary Data are available at www.liebertpub.com/thy).

\section{Thyroid volume in subjects with hemiagenesis}

Multivariate regression analysis demonstrated that sex, age, body surface area, and hemiagenesis independently affected the remaining hemithyroid volume (Table 2). Age was the strongest factor affecting the hemithyroid volume among the four factors studied. In addition, the sex- and age-specific body surface area independently affected the hemithyroid volume in either right or left hemiagenesis.

To compare the intact thyroid volume between subjects with hemiagenesis and normal subjects, ANCOVA was performed after adjusting for body surface area. As shown in Table 3, the intact thyroid volumes in subjects with hemiagenesis were significantly larger than those of the ipsilateral lobes in normal subjects after correcting for body surface area. When the same analysis was performed between male and female subjects and among four age groups, the results were similar to those obtained from the entire cohort.

\section{Discussion}

This is the first in-depth survey of thyroid hemiagenesis and calculation of reference values for thyroid volume by systematic ultrasound examinations of approximately 300,000 subjects aged 0-23 years in Japan.

All subjects were screened for bilateral thyroid agenesis by performing an ultrasound examination. Subjects with an ectopic thyroid may have been included among those in whom thyroid agenesis was detected. Thus, the actual number of subjects with complete thyroid tissue defects may be lower than the results of the present study show. The reported overall prevalence of congenital hypothyroidism ranges from $0.025 \%$ to $0.050 \%$ (9), and the reported prevalence of congenital hypothyroidism in Japan is $0.028 \%$ (1/3472) (9). Approximately $80-90 \%$ of reported cases are due to developmental defects of the thyroid gland (10). Thus, using the findings of the two aforementioned studies, the calculated prevalence of thyroid defects is $0.023-0.026 \%$. However, the prevalence of thyroid agenesis in the present study was lower than that in previous reports. Because this study was based on a voluntary survey and thyroid agenesis is symptomatic (in contrast to hemiagenesis, which is asymptomatic), screening bias is a possible explanation for the low prevalence of thyroid agenesis. Additionally, thyroid hormone replacement may have resulted in shrinkage of the residual thyroid gland in some subjects with agenesis.

Several reports have determined the prevalence of thyroid hemiagenesis outside Japan. According to a study of an area endemic for goiter, 16/71,500 $(0.022 \%)$ subjects reportedly

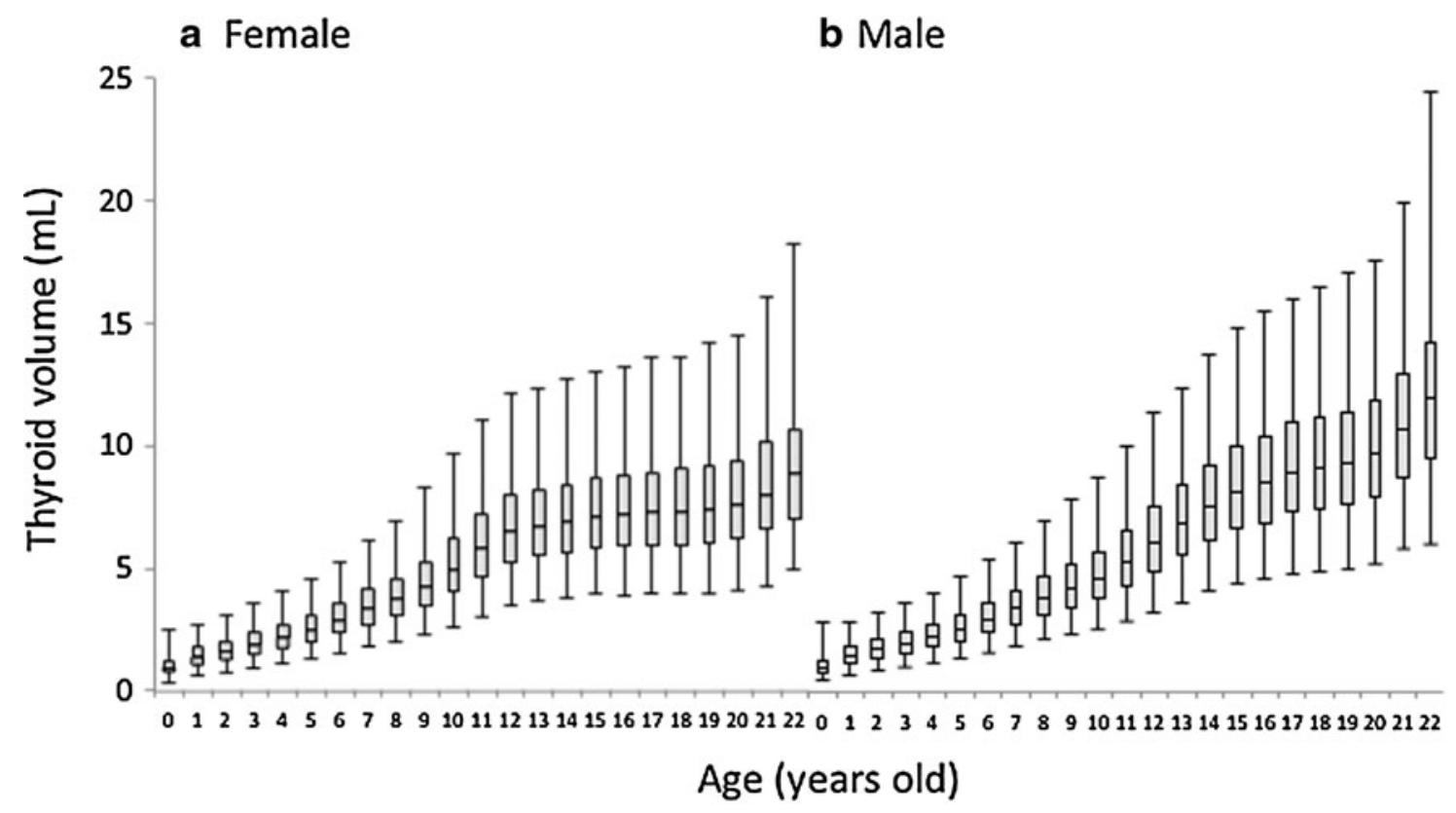

FIG. 1. Box-and-whisker plots showing distributions of thyroid volume according to age and sex. The width, thickness, and height of each lobe were measured, and the volume of each lobe was calculated by the mean elliptical shape volume, determined as follows: $\pi / 6 \times$ width $\times$ thickness $\times$ height. The sum of the volume of both lobes was the thyroid volume. The boxes represent the 25th and 75th percentiles, and the horizontal lines within the box represent the median values. The whiskers represent the 2.5th and 97.5th percentiles. Each value was plotted by age in (a) female and (b) male subjects. 
Table 2. Association of Left (Upper Panel) and Right (Lower Panel) Hemithyroid Volume with Sex, Age, SeX- And Age-Specific Body Surface Area, and Hemiagenesis

\begin{tabular}{|c|c|c|}
\hline Factors & Volume, mL [CI] & $\mathrm{p}$-Value \\
\hline \multicolumn{3}{|l|}{ Left thyroid } \\
\hline Sex (male vs. female) & $0.06[0.055-0.069]$ & $<0.001$ \\
\hline Age (per $1 S D$ increase) ${ }^{\mathrm{a}}$ & $1.05[1.046-1.053]$ & $<0.001$ \\
\hline 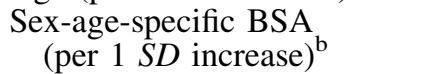 & $0.34[0.340-0.347]$ & $<0.001$ \\
\hline $\begin{array}{l}\text { Right hemiagenesis } \\
\text { (bilateral vs. hemiagenesis) }\end{array}$ & $1.38[0.841-1.924]$ & $<0.001$ \\
\hline \multicolumn{3}{|l|}{ Right thyroid } \\
\hline Sex (male vs. female) & $0.04[0.029-0.045]$ & $<0.001$ \\
\hline Age $(\text { per } 1 S D)^{\mathrm{a}}$ & $1.28[1.279-1.287]$ & $<0.001$ \\
\hline $\begin{array}{l}\text { Sex-age-specific BSA } \\
\quad(\text { per } 1 S D)^{\mathrm{b}}\end{array}$ & $0.43[0.430-0.438]$ & $<0.001$ \\
\hline $\begin{array}{l}\text { Left hemiagenesis } \\
\text { (bilateral vs. hemiagenesis) }\end{array}$ & 1.70 [1.399-1.991] & $<0.001$ \\
\hline
\end{tabular}

${ }^{\mathrm{a}} 1 S D: 5.0$ years; ${ }^{\mathrm{b}} 1 S D: 0.15 \mathrm{~m}^{2}$

CI, confidence interval; SD, standard deviation; BSA, body surface area.

had thyroid hemiagenesis (11). In the same study, a large meta-analysis revealed an estimated $0.05 \%$ prevalence of thyroid hemiagenesis. There was a left-to-right ratio of thyroid hemiagenesis of 3.6:1.0, with an isthmus present in 44\%, and the female:male ratio was 3.0:1.0.

Among unselected 11- to 14-year-old schoolchildren from southeastern Sicily, 12/24,032 (0.05\%) subjects reportedly had thyroid hemiagenesis. In that study, the female:male ratio was 1.0:1.4, and thyroid hemiagenesis developed in the left lobe in all subjects (12). Furthermore, in a study of 4004 asymptomatic schoolchildren in the iodine-sufficient area of northern Poland, two $(0.05 \%)$ subjects were found to have thyroid hemiagenesis (13). In a Turkish study of a sample of the general population, the prevalence of thyroid hemiagenesis detected using ultrasonographic screening was $0.25 \%$. (14). A separate Turkish study of 6242 subjects reported $10(0.16 \%)$ cases of hemiagenesis ( 8 females), although all subjects were outpatients with various disorders (15). In Japan, two $(0.05 \%)$ cases were reported among 4365 schoolchildren volunteering in an ultrasonographic study (16). Taken together, the findings of the aforementioned reports demonstrate that the prevalence of thyroid hemiagenesis is approximately $0.02-0.05 \%$ in normal subjects. The reported prevalence, sex ratio, and laterality are summarized in Table 4.

In the present study, 151,374 male and 148,534 female subjects were investigated, in whom hemiagenesis was detected in $0.016 \%$ and $0.027 \%$, respectively. Although the difference was not significant, female subjects were predominantly affected, indicating that the prevalence of and sex-related difference in hemiagenesis are in agreement with previous reports. However, no sex-related difference in left hemiagenesis was observed in the present study. The significant sex-related difference observed in right hemiagenesis may indicate the contribution of sex-related factor(s) to the development of right but not left hemiagenesis. A significant female predominance was observed in the development of ectopic thyroid glands but not in athyreosis (17). Genetic factors in thyroid development may affect the predominance

Table 3. Thyroid Volume Assessment in Total Cohort, Each Sex, and Four Age Groups After Adjustment for Sex, Age, and Sex-Age-Specific Body Surface Area

\begin{tabular}{|c|c|c|c|c|c|}
\hline & Normal controls $(m L)$ & $\mathrm{n}$ & Hemiagenesis $(\mathrm{mL})$ & $\mathrm{n}$ & $\mathrm{p}$ \\
\hline $\begin{array}{l}\text { All } \\
\text { Right } \\
\text { Left }\end{array}$ & $\begin{array}{l}3.06 \pm 1.08^{\mathrm{a}} \\
2.52 \pm 1.08\end{array}$ & $\begin{array}{l}292,398 \\
292,440\end{array}$ & $\begin{array}{l}4.75 \pm 1.10^{\mathrm{b}} \\
3.90 \pm 0.97^{\mathrm{c}}\end{array}$ & $\begin{array}{l}54 \\
12\end{array}$ & $\begin{array}{l}<0.001 \\
<0.001\end{array}$ \\
\hline $\begin{array}{l}\text { Female } \\
\text { Right } \\
\text { Left }\end{array}$ & $\begin{array}{l}3.00 \pm 1.14 \\
2.49 \pm 1.14\end{array}$ & $\begin{array}{l}144,847 \\
144,867\end{array}$ & $\begin{array}{l}4.94 \pm 1.10 \\
4.30 \pm 0.95\end{array}$ & $\begin{array}{l}30 \\
10\end{array}$ & $\begin{array}{l}<0.001 \\
<0.001\end{array}$ \\
\hline $\begin{array}{l}\text { Male } \\
\text { Right } \\
\text { Left }\end{array}$ & $\begin{array}{l}3.12 \pm 1.15 \\
2.55 \pm 0.77\end{array}$ & $\begin{array}{l}147,551 \\
147,573\end{array}$ & $\begin{array}{l}4.54 \pm 1.08 \\
2.01 \pm 0.95\end{array}$ & $\begin{array}{r}24 \\
2\end{array}$ & $<{ }_{\mathrm{d}}^{<0.001}$ \\
\hline $\begin{array}{l}\text { 0-6 years } \\
\text { Right } \\
\text { Left }\end{array}$ & $\begin{array}{l}1.29 \pm 0.52 \\
1.10 \pm 0.52\end{array}$ & $\begin{array}{l}68,249 \\
68,268\end{array}$ & $\begin{array}{l}1.80 \pm 0.46 \\
1.85 \pm 0.40\end{array}$ & $\begin{array}{r}21 \\
2\end{array}$ & $\begin{array}{c}<0.001 \\
-\end{array}$ \\
\hline $\begin{array}{l}\text { 7-11 years } \\
\text { Right } \\
\text { Left }\end{array}$ & $\begin{array}{l}2.53 \pm 0.89 \\
2.06 \pm 0.59\end{array}$ & $\begin{array}{l}88,338 \\
88,345\end{array}$ & $\begin{array}{l}4.23 \pm 0.82 \\
3.98 \pm 0.73\end{array}$ & $\begin{array}{r}10 \\
3\end{array}$ & $\begin{array}{l}<0.001 \\
<0.001\end{array}$ \\
\hline $\begin{array}{l}\text { 12-16 years } \\
\text { Right } \\
\text { Left }\end{array}$ & $\begin{array}{l}4.06 \pm 1.18 \\
3.32 \pm 1.18\end{array}$ & $\begin{array}{l}87,706 \\
87,717\end{array}$ & $\begin{array}{l}5.82 \pm 1.32 \\
5.54 \pm 1.14\end{array}$ & $\begin{array}{r}16 \\
5\end{array}$ & $\begin{array}{l}<0.001 \\
<0.001\end{array}$ \\
\hline $\begin{array}{l}\text { 17-23 years } \\
\text { Right } \\
\text { Left }\end{array}$ & $\begin{array}{l}4.72 \pm 1.54 \\
3.91 \pm 1.32\end{array}$ & $\begin{array}{l}48,105 \\
48,110\end{array}$ & $\begin{array}{l}9.82 \pm 1.59 \\
3.11 \pm 1.39\end{array}$ & $\begin{array}{l}7 \\
2\end{array}$ & $\begin{array}{c}<0.001 \\
-\end{array}$ \\
\hline
\end{tabular}

${ }^{\mathrm{a}}$ Data are shown as mean \pm standard deviation.

${ }^{\mathrm{b}}$ Values were obtained from subjects with left hemiagenesis.

${ }^{\mathrm{c}}$ Values were obtained from subjects with right hemiagenesis.

${ }^{\mathrm{d}}$ Because the sample number was $<3$, the $p$-value was not calculated. 
Table 4. Overview of Subjects with Thyroid Hemiagenesis in Previous Studies and the Present Study

\begin{tabular}{|c|c|c|c|c|c|c|c|}
\hline Subjects & Location & $\begin{array}{l}\text { Total } \\
\text { number } \\
\text { (n) }\end{array}$ & $\begin{array}{l}\text { Hemiagenesis } \\
\text { (n) }\end{array}$ & $\begin{array}{c}\text { Prevalence } \\
(\%)\end{array}$ & $\begin{array}{l}\text { Female: } \\
\text { male ratio }\end{array}$ & $\begin{array}{l}\text { Left: } \\
\text { right ratio }\end{array}$ & Reference \\
\hline $\begin{array}{l}\text { Patients with } \\
\text { goiter }\end{array}$ & $\begin{array}{l}\text { Austria } \\
\text { and } \\
\text { Carinthia }\end{array}$ & 71,500 & 16 & 0.02 & 1.33 & 15 (1 right hemiagenesis) & (11) \\
\hline Meta-analysis $^{\mathrm{a}}$ & $\begin{array}{l}\text { Various } \\
\text { places }\end{array}$ & 226,117 & 119 & 0.05 & NA & NA & (11) \\
\hline Aged $11-14$ years & Sicily, Italy & 24,032 & 12 & 0.05 & 0.71 & All left hemiagenesis & (12) \\
\hline Schoolchildren & $\begin{array}{l}\text { Northern } \\
\text { Poland }\end{array}$ & 4004 & 2 & 0.05 & $\begin{array}{c}\text { Female } \\
\text { in all cases }\end{array}$ & All left hemiagenesis & (13) \\
\hline Thyroid disease & Turkey & 4833 & 12 & 0.25 & 0.64 & All left hemiagenesis & (14) \\
\hline Thyroid disease & Turkey & 6242 & 10 & 0.16 & NA & 2.33 & (15) \\
\hline Schoolchildren & Japan & 4365 & 2 & 0.05 & NA & NA & (16) \\
\hline Aged $0-22$ years & Japan & 299,908 & 67 & 0.02 & 1.67 & 4.58 & $\begin{array}{l}\text { Present } \\
\text { study }\end{array}$ \\
\hline
\end{tabular}

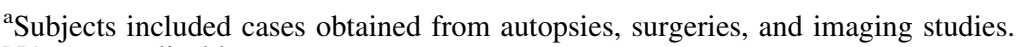

NA, not applicable.

of thyroid dysgenesis, especially the development of hemiagenesis and ectopic thyroid in females. The laterality of abnormal thyroid development may be affected by some sexrelated factors, similar to the development of ectopic thyroid.

The thyroid volume in Japanese children and young adults who underwent thyroid ultrasound examination as the initial preliminary survey from October 9, 2011, to March 31, 2012, was previously reported (7). The current study reports the thyroid volumes of a total of 292,428 children and young adults, including the 2.5 th and 97.5 th percentiles of the thyroid volumes of subjects aged 20-22 years whose body surface area was 1.9 and $2.0 \mathrm{~m}^{2}$ in females and $2.3 \mathrm{~m}^{2}$ in males. Both iodine deficiency and excess result in an increased thyroid volume, although measurement of the thyroid volume with ultrasound should be standardized to improve comparability (18). The reported median urinary iodine concentrations in Fukushima Prefecture were $180.5 \mu \mathrm{g} / \mathrm{L}$ in the Hamadori area and $212.0 \mu \mathrm{g} / \mathrm{L}$ in the Nakadori and Aizu area (19), indicating that the iodine intake is not at low levels that may affect thyroid volume.

Because thyroid hemiagenesis is suspected to be congenital, the contralateral thyroid is thought to be stimulated by thyrotropin through a negative feedback mechanism that compensates for the unilateral thyroid defect. Thus, subclinical hypothyroidism may induce enlargement of the thyroid gland in some cases. As expected, the presence of hemiagenesis independently increased the volume of the intact thyroid lobe among the factors of sex, age, and body surface area. ANCOVA also showed that the intact lobes of the thyroid in subjects with hemiagenesis were larger than the ipsilateral lobes in normal subjects. These results were consistent when the calculations were performed according to sex and age group. Because only two subjects had hemiagenesis in the male group and the 17- to 23-year-old age group, a statistical analysis was not performed. All statistical analyses were performed after correction for body surface area in terms of the relationship between thyroid volume and hemiagenesis. Notably, hemiagenesis itself may cause changes in body surface area because the thyroid volume was associated with the body surface area in the present study and the preliminary survey (7). To the best of the authors' knowledge, this is the first report to provide statistical evidence that an intact thyroid lobe in patients with hemiagenesis is larger than a healthy ipsilateral thyroid lobe.

In conclusion, the present study revealed that the prevalence of thyroid hemiagenesis is $0.02 \%$, with a female:male ratio of 1.67:1.00. These data are equivalent to those previously reported. It is possible that sex affects thyroid morphogenesis, especially in terms of lateral development of the gland, although further investigation is needed to confirm this.

\section{Acknowledgments}

We express our gratitude to all subjects who participated in the Fukushima Health Management Survey. We also thank Ms. Tazuko Kawasaki and Ms. Takako Takahashi for their excellent secretarial assistance. This survey was conducted as part of Fukushima Prefecture's post-disaster recovery plans and was supported by the national "Health Fund for Children and Adults Affected by the Nuclear Incident." The findings and conclusions of this article are solely the responsibility of the authors and do not represent the official views of the Fukushima Prefecture government.

Other participating expert committee members, advisors, and staff members in the Fukushima Health Management Survey: Kenji Kamiya, Seiji Yasumura, Kenneth E. Nollet, Kumiko Tsuboi, Shiro Matsui, Seisho Tanaka, Masaharu Maeda, Shigeatsu Hashimoto, Keiya Fujimori, Suguru Ishida, Testuo Ishikawa, Akira, Sakai, Yuko Hino, Hiroshi Mizunuma, Keiichi Nakano, Satoshi Suzuki, Chiyo Ohkouchi, Tomomi Hakoiwa, Chisato Takahashi, Yukari Sato, Ayako Sato, Nobuko Sakuma, Toshie Sakagami, Manabu Ohishi, Norikazu Abe, Masahiko Henmi, Yukie Yamaya, Takao Yamahata, Yukiko Horikoshi, Yoko Nihei, Risa Ujiie, Sakiko Meguro, Ayana Okazaki, Yumiko Kurozu, Mizuki Sekino, Yuko Sato, and Yayoi Sato.

\section{Author Disclosure Statement}

The authors have nothing to disclose. There is no conflict of interest in this study. 


\section{References}

1. Henle J 1986 Hedbuch der Systematischen Anatomie des Menschen. Friedrich Viewig und Sohn, Braunschweig, Germany.

2. Melnick JC, Stemkowski PE 1981 Thyroid hemiagenesis (hockey stick sign): a review of the world literature and a report of four cases. J Clin Endocrinol Metab 52:247.

3. Shabana W, Delange F, Freson M, Osteaux M, De Schepper J 2000 Prevalence of thyroid hemiagenesis: ultrasound screening in normal children. Eur J Pediatr 159:456-458.

4. UNSCEAR 2013 Sources, effects and risks of ionizing radiation. Volume I Annex A: Levels and effects of radiation exposure due to the nuclear accident after the 2011 Great East-Japan Earthquake and Tsunami. Available at: www.unscear.org/unscear/en/publications/2013/UNSCEAR_ 2013_Report_Vol.1.pdf (accessed December 14, 2016).

5. Jacob P, Kenigsberg Y, Zvonova I, Goulko G, Buglova E, Heidenreich WF, Golovneva A, Bratilova AA, Drozdovitch V, Kruk J, Pochtennaia GT, Balonov M, Demidchik EP, Paretzke HG 1999 Childhood exposure due to the Chernobyl accident and thyroid cancer risk in contaminated areas of Belarus and Russia. Br J Cancer 80:1461-1469.

6. Suzuki S, Suzuki S, Fukushima T, Midorikawa S, Shimura H, Matsuzuka T, Ishikawa T, Takahashi H, Ohtsuru A, Sakai A, Hosoya M, Yasumura S, Nollet K, Ohira T, Ohto H, Abe M, Kamiya K, Yamashita S 2016 Comprehensive survey results of childhood thyroid ultrasound examinations in Fukushima in the first four years after the Fukushima Daiichi Nuclear Power Plant accident. Thyroid 26: 843-851.

7. Suzuki S, Midorikawa S, Fukushima T, Shimura H, Ohira T, Ohtsuru A, Abe M, Shibata Y, Yamashita S, Suzuki S 2015 Systematic determination of thyroid volume by ultrasound examination from infancy to adolescence in Japan: the Fukushima Health Management Survey. Endocr J 62:261-268.

8. Dubois D, Dubois EF 1961 A formula to estimate the approximate surface area if height and weight be known. Arch Intern Med 17:863-871.

9. Ford G, LaFranchi SH 2014 Screening for congenital hypothyroidisim: a worldwide view of strategies. Best Pract Res Clin Endocrinol Metab 28:175-187.

10. Van Vliet G 2001 Treatment of congenital hypothyroidism. Lancet 358:86-87.

11. Mikosch P, Gallowitsch HJ, Kresnik E, Molnar M, Gomez I, Lind P 1999 Thyroid hemiagenesis in an endemic goiter area diagnosed by ultrasonography: report of sixteen patients. Thyroid 9:1075-1084.

12. Maiorana R, Carta A, Floriddia G, Leonardi D, Buscema M, Sava L, Calaciura F, Vigneri R 2003 Thyroid hemi- agenesis: prevalence in normal children and effect on thyroid function. J Clin Endocrinol Metab 88:1534-1536.

13. Korpal-Szczyrska M, Kosiak W, Swieton D 2008 Prevalence of thyroid hemiagenesis in an asymptomatic schoolchildren population. Thyroid 18:637-639.

14. Gursoy A, Anil C, Unal AD, Demirer AN, Tutuncu NB, Erdogan MF 2008 Clinical and epidemiological characteristics of thyroid hemiagenesis: ultrasound screening in patients with thyroid disease and normal population. Endocrine 33:338-341.

15. Berker D, Ozuguz U, Isik S, Aydin Y, Ates Tutuncu Y, Akbaba G, Guler S 2010 A report of ten patients with thyroid hemiagenesis: ultrasound screening in patients with thyroid disease. Swiss Med Wkly 140:118-121.

16. Hayashida N, Imaizumi M, Shimura H, Okubo N, Asari Y, Nigawara T, Midorikawa S, Kotani K, Nakaji S, Otsuru A, Akamizu T, Kitaoka M, Suzuki S, Taniguchi N, Yamashita S, Takamura N 2013 Investigation Committee for the Proportion of Thyroid Ultrasound Findings. Thyroid ultrasound findings in children from three Japanese prefectures: Aomori, Yamanashi and Bagasaki. PLoS One 8:e83220.

17. Devos H, Rodd C, Gagne N, Laframboise R, Van Vliet G 1999 A search for the possible molecular mechanisms of thyroid dysgenesis: sex ratios and associated malformations. J Clin Endocrinol Metab 84:2502-2506.

18. Zimmermann MB, Hess SY, Molinari L, De Benoist B, Delange F, Braverman LE, Fujieda K, Ito Y, Jooste PL, Moosa K, Pearce EN, Pretell EA, Shishiba Y 2004 New reference values for thyroid volume by ultrasound in iodine-sufficient schoolchildren: a World Health Organization/Nutrition for Health and Development Iodine Deficiency Study Group Report. Am J Clin Nutr 79:231-237.

19. Tsubokura M, Nomura S, Watanabe H, Nishikawa Y, Suzuki C, Ochi S, Leppold C, Kinoshita H, Kato S, Saito Y 2016 Assessment of nutritional status of iodine through urinary iodine screening among local children and adolescents after the Fukushima Daiichi Nuclear Power Plant Accident. Thyroid 26:1778-1785.

Address correspondence to: Satoru Suzuki, MD, PhD

Department of Thyroid and Endocrinology Division of Internal Medicine Fukushima Medical University Hospital 1 Hikarigaoka Fukushima City Fukushima 960-1295 Japan

E-mail: suzukisa@fmu.ac.jp 